\title{
Javelin diagrams: applications in veterinary medical decision analysis
}

\author{
Johann C. DETILLEUX* \\ Department of Animal Production, Faculty of Veterinary Medicine, Liège University, \\ Boulevard de Colonster, 4000 Liège, Belgium
}

(Received 22 January 2004; accepted 28 April 2004)

\begin{abstract}
This paper introduces javelin diagrams as an innovative way for depicting the results of medical decision analyses. The methods were used to determine whether, and at which values, blood lactate in Belgian White and Blue or maximum tidal volumes in Holstein calves should be measured before deciding to treat or not a calf suffering from the bovine respiratory disease complex. The different alternatives depended upon the probabilities of survival with and without treatment and upon the costs associated with a possible death, the test and the treatment. The chosen alternative was the one with the lowest expected costs. From data collected on the treated calves, the expected costs of measuring lactate $(198.01 €)$ and tidal volumes $(27.38 €)$ before deciding to treat or not were lower than the expected costs of directly treating sick Belgian Blue (215.39 €) and Holstein (51.55€) calves, respectively. The treatment should be applied to sick Belgian Blue calves with blood lactate $\leq 7.8 \mathrm{mmol} / \mathrm{L}$ and to Holstein calves with a maximum tidal volume $\geq 1.81 \mathrm{~L}$. At such test values, the treatment expected costs were lower than the expected costs associated with no treatment of calves with other test values. Probabilistic sensitivity analyses showing benefits in treating animals with a positive test (over not treating the animals with a negative test) were mostly invariant to changes in any cost value but were sensitive to uncertainties in probabilities of survival with or without treatment. The javelin diagrams provided a clear visual indication of such results. They depicted how and by how much the benefits were affected by uncertainties in probabilities, they proposed different information values associated with the chosen alternative, and identified directions for further research.
\end{abstract}

clinical decision analysis / javelin diagrams / bovine respiratory disease complex

\section{INTRODUCTION}

After they have reached their diagnosis, veterinarians must decide whether or not to treat a patient. Treatment may be indicated when patients with advanced disease are at risk of mortality in the face of continuous disease progression. Conversely, treatment may be withheld when its costs outweigh its benefits. Veterinarians may perform a clinical test to help them reach their decision. They will decide to gather this additional information if the expected cost of performing the test is lower than the expected cost of directly treating the disease. At the very least, this decision will depend upon the sensitivity and specificity of the test in evaluating survival (the veterinarian will be

\footnotetext{
* Corresponding author: jdetilleux@ulg.ac.be
} 
more confident in a test accurate in identifying surviving animals), and upon the treatment (the veterinarian may be more ready to perform the test if the treatment is very expensive) or test costs (the veterinarian will be less willing to do the test if it is expensive). When the test is quantitative, it is also necessary to define cut-off values to distinguish between animals for which doing the treatment is economically beneficial from those for which not doing the treatment is advantageous.

Decision analysis methods provide a formal framework to chose between different alternatives: they quantify and combine in an explicit way the information provided and recommend to choose the alternative which maximizes the expected value (or minimizes the expected cost) among possible alternatives $[10,11]$. Therefore, they are particularly adequate for determining whether or not a clinical test should be performed, and at which test value the treatment should be executed such that the chosen alternative is the least costly.

Uncertainty is inherent in such decision and the chosen alternative may change because sensitivities and specificities of the test are usually observed on a small sample and because knowledge of some variables, such as costs and probabilities of survival, is incomplete. A mathematical way to deal with such uncertainties is to perform probabilistic sensitivity analyses by providing prior probability distributions to the variables and to compute post-hoc probability distributions to the chosen alternative [8]. Recently, Felli and Hazen [6] proposed to present the results of such sensitivity analyses as javelin diagrams that display both the robustness of the results (the degree to which they fluctuate against deviations in the input variables) and the information value associated with the decision (the expected gain in getting more information on an uncertain input variable).

The objective of this paper was to introduce javelin diagrams in veterinary medical decision analyses. The analyses consisted in determining whether or not the expected costs of performing a clinical test before deciding on treatment were lower than the expected costs of directly treating a patient, and at which test values treating it was less expensive than not treating it. The best alternative was chosen according to the patient survival rates, costs of treatment and test, and possible patient death. Data were from studies on blood lactate and tidal volumes in calves treated for the bovine respiratory disease complex (BRDC). After choosing an alternative, sensitivity analyses were performed to determine the robustness of the estimates obtained with the decision analyses and the results are depicted in the javelin diagrams.

\section{MATERIALS AND METHODS}

\subsection{Clinical studies of the bovine respiratory disease complex}

Respiratory disease may challenge the oxygen transport chain in different ways, e.g., through reduced oxygen transfer from the lungs to the arterial blood or an elevated oxygen consumption due to an increased work of breathing. Therefore, studies were undertaken by the Department of Physiology of the Veterinary Faculty of Liège (Belgium) in order to determine whether the dosages of blood lactate (indirect assessment of the oxygen level in tissues) and tidal volume (lung function test) in calves suffering from BRDC could be an index of disease severity. The BRDC was diagnosed by veterinarians and indicated by laborious breathing associated with either cough, nasal discharge, fever, anorexia or adventitious lung sounds on auscultation.

In the first clinical study, 96 Belgian White and Blue calves, all diagnosed with BRDC, were followed from 28 days to 13 months of age [3]. At the start of the follow-up period, the veterinarians sampled and treated plasma lactate (PL) levels on each calf. Treatment was standardized according to the literature on the efficacy of known 
therapeutics and clinical signs such as rectal temperature, respiratory rate, and partial arterial oxygen pressure. During the follow-up period, 74 calves recovered after a treatment and 22 died. The treatment costs averaged $25.94 \pm 6.71 €$ and $52.5 \pm 19.2 €$ among recovering and incurable calves, respectively. Venous blood lactate was analyzed immediately with a portable lactate analyzer at a cost of $1.75 €$. The PL values increased from an average of $1.78 \pm$ $0.10 \mathrm{mmol} / \mathrm{L}$ among calves recovering with treatment up to $10.61 \pm 1.21 \mathrm{mmol} / \mathrm{L}$ among calves dying even after intensive treatment. The calves were valued at around $800 \pm 200 €$.

In the second study, veterinarians measured the maximum tidal volumes (TV) on 249 Holstein calves, after injection of a respiratory analeptic ([1], unpublished data). Each calf respiratory flow was measured with a heated pneumotachograph and the TV were recorded with a digital acquisition analysis program at a cost of $25 €$ (see [1] for more technical details). The veterinarians measured each calf, at the start of the 6-month survey, when calves presented no clinical signs of BRDC. A total of 86 calves developed the disease for which they were all treated but seven of them died. The values for TV averaged $1.41 \pm 0.05 \mathrm{~L}$ and $1.19 \pm$ $0.14 \mathrm{~L}$ in calves recovering or not from the disease. The choice of medicine was left to the veterinarians' judgment and treatment costs were obtained for each calf. They averaged $40.24 \pm 3.13 €$ among recovering calves and $79.25 \pm 20.04 €$ among dead calves, not including the calves' economic values, estimated at $100 \pm 25 €$.

\subsection{Analyses}

To illustrate the worth of the javelin diagrams, two questions were raised from the information collected in both clinical studies. The first question was whether it would have been economical to do the clinical test before the treatment, i.e., whether the expected costs of treating or not an animal based on its test results $\left(=\mathrm{EC}_{\mathrm{TEST}}\right)$ were lower than the expected costs of a treatment without a test $\left(=\mathrm{EC}_{\mathrm{TRT}}\right)$. Since the values for PL and $\mathrm{TV}$ are continuous, the individual $\mathrm{EC}_{\mathrm{TEST}}$ were first computed at each observed value of PL (from 1 to $15 \mathrm{mmol} / \mathrm{L}$ ) and TV (from 0.66 to $3.08 \mathrm{~L}$ ). Then, the overall $\mathrm{EC}_{\mathrm{TEST}}$ was calculated as the weighted average of all individual $\mathrm{EC}_{\mathrm{TEST}}$, weighted by the frequency of occurrence of each test value.

The second issue was to identify PL and TV cut-off values at which the expected costs of treating a calf with a positive test result $\left(=\mathrm{EC}_{\mathrm{TP}}\right)$ were lower than the expected costs of not treating a calf with a negative test result $\left(=\mathrm{EC}_{\mathrm{TN}}\right)$ or, equivalently, at which $\mathrm{EC}_{\mathrm{DIFF}}=\mathrm{EC}_{\mathrm{TN}}-\mathrm{EC}_{\mathrm{TP}}$ was positive. Under the assumption that veterinarians would treat only sick calves with positive results, i.e., calves with test values above (or below) some threshold to be defined, the four expected costs were computed as:

$$
\begin{aligned}
\mathrm{EC}_{\mathrm{TRT}}= & \mathrm{p}(\mathrm{S}+) \times \mathrm{C}_{\mathrm{MILD}}+[1-\mathrm{p}(\mathrm{S}+)] \\
& \times\left(\mathrm{C}_{\mathrm{ANL}}+\mathrm{C}_{\mathrm{SEVERE}}\right) \\
\mathrm{EC}_{\mathrm{TEST}}= & \mathrm{C}_{\mathrm{TEST}}+\mathrm{p}(\mathrm{S}+\cap \mathrm{T}+) \times \mathrm{C}_{\mathrm{MILD}} \\
& +\mathrm{p}(\mathrm{S}-\cap \mathrm{T}+) \times\left(\mathrm{C}_{\mathrm{SEVERE}}\right) \\
& +\mathrm{p}(\mathrm{S}-) \times \mathrm{C}_{\mathrm{ANL}} \\
\mathrm{EC}_{\mathrm{TP}}= & \mathrm{p}(\mathrm{T}+) \times \mathrm{C}_{\mathrm{TEST}} \\
& +\mathrm{p}(\mathrm{S}+\cap \mathrm{T}+) \times \mathrm{C}_{\mathrm{MILD}} \\
& +\mathrm{p}(\mathrm{S}-\cap \mathrm{T}+) \times\left(\mathrm{C}_{\mathrm{SEVERE}}+\mathrm{C}_{\mathrm{ANL}}\right)
\end{aligned}
$$

$\mathrm{EC}_{\mathrm{TN}}=\mathrm{p}(\mathrm{T}-) \times \mathrm{C}_{\mathrm{TEST}}+\mathrm{p}(\mathrm{S}-\cap \mathrm{T}-) \times \mathrm{C}_{\mathrm{ANL}}$

where the input variables are: $\mathrm{C}_{\mathrm{ANL}}$ for the economic loss due to the patient's death, $\mathrm{C}_{\text {MILD }}$ for the treatment cost of a recovering patient, $\mathrm{C}_{\text {SEVERE }}$ for the treatment cost of an incurable case, $\mathrm{C}_{\text {TEST }}$ for the test cost, $\mathrm{p}(\mathrm{S}+)$ for the probability of survival, $\mathrm{p}(\mathrm{S}+\cap \mathrm{T}+)$ for the probability of survival with a positive test, and $\mathrm{p}(\mathrm{S}-\cap \mathrm{T}+)$ for the probability of death with a positive test.

After having answered both matters, we performed probabilistic sensitivity analyses to determine how $\mathrm{EC}_{\mathrm{DIFF}}$ varied with uncertainties in the input variables. Indeed, probabilities of survival were biased in our 

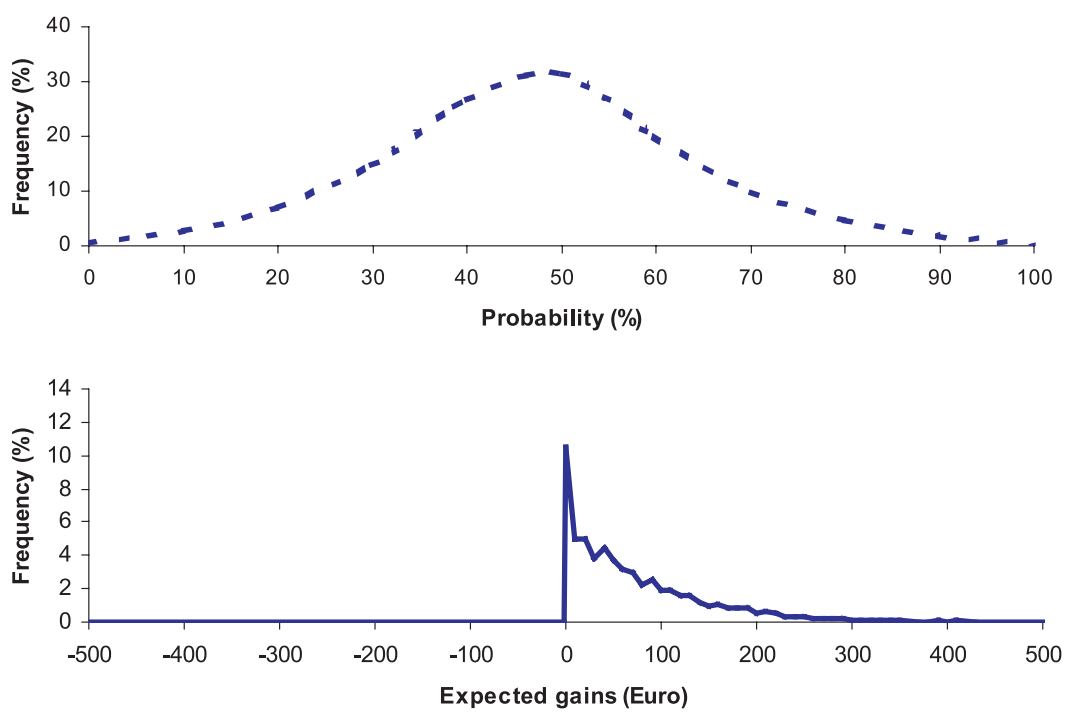

Figure 1. Javelin diagram representing the hypothetical variation in an output variable (plain line) associated with a hypothetical distribution of a input variable (dashed line).

Table I. Mean (standard deviation) of the distribution of the input variables used in the decision analyses.

\begin{tabular}{lcc}
\hline Input variables & Blood lactate & Tidal volume \\
\hline Probability of survival & $0.77(0.42)$ & $0.97(0.16)$ \\
Probability of a negative test result & $0.13(0.34)$ & $0.17(0.37)$ \\
Probability of a death with a negative test result & $0.13(0.34)$ & $0.03(0.18)$ \\
Probability of survival with a positive test result & $0.77(0.42)$ & $0.17(0.37)$ \\
Cost of treatment for a surviving patient $(€)$ & $25.94(6.7)$ & $40.24(3.13)$ \\
Cost of treatment for a fatal case $(€)$ & $52.5(18)$ & $79.25(20.04)$ \\
Animal economic value $(€)$ & $800(65)$ & $100(25)$ \\
\hline
\end{tabular}

studies since no information on spontaneous cure was available. Uncertainties in the probabilities and costs are represented by beta and normal distributions, respectively (with the exception of the test cost known with certainty). The location and dispersion parameters for both types of distributions were set equal to their observed values (Tab. I) and Monte Carlo simulation was used to generate the distribution of $\mathrm{EC}_{\mathrm{DIFF}}$. The results are depicted as javelin diagrams (Fig. 1). The expected costs were computed with the matrix language SAS/IML [9], either for each input distribution separately (to obtain six javelin diagrams, one per input variable, with the other variables fixed at their observed value) or collectively over the six distributions (to have one overall diagram for all variables). In either case, the left and right extremes of the javelin represent the lowest and highest $\mathrm{EC}_{\mathrm{DIFF}}$ values and relate to values in the lower and upper tails of the input distributions. The head of the javelin represents the positive part of the distribution of $\mathrm{EC}_{\mathrm{DIFF}}$. Only the positive part needs to be shown because it 


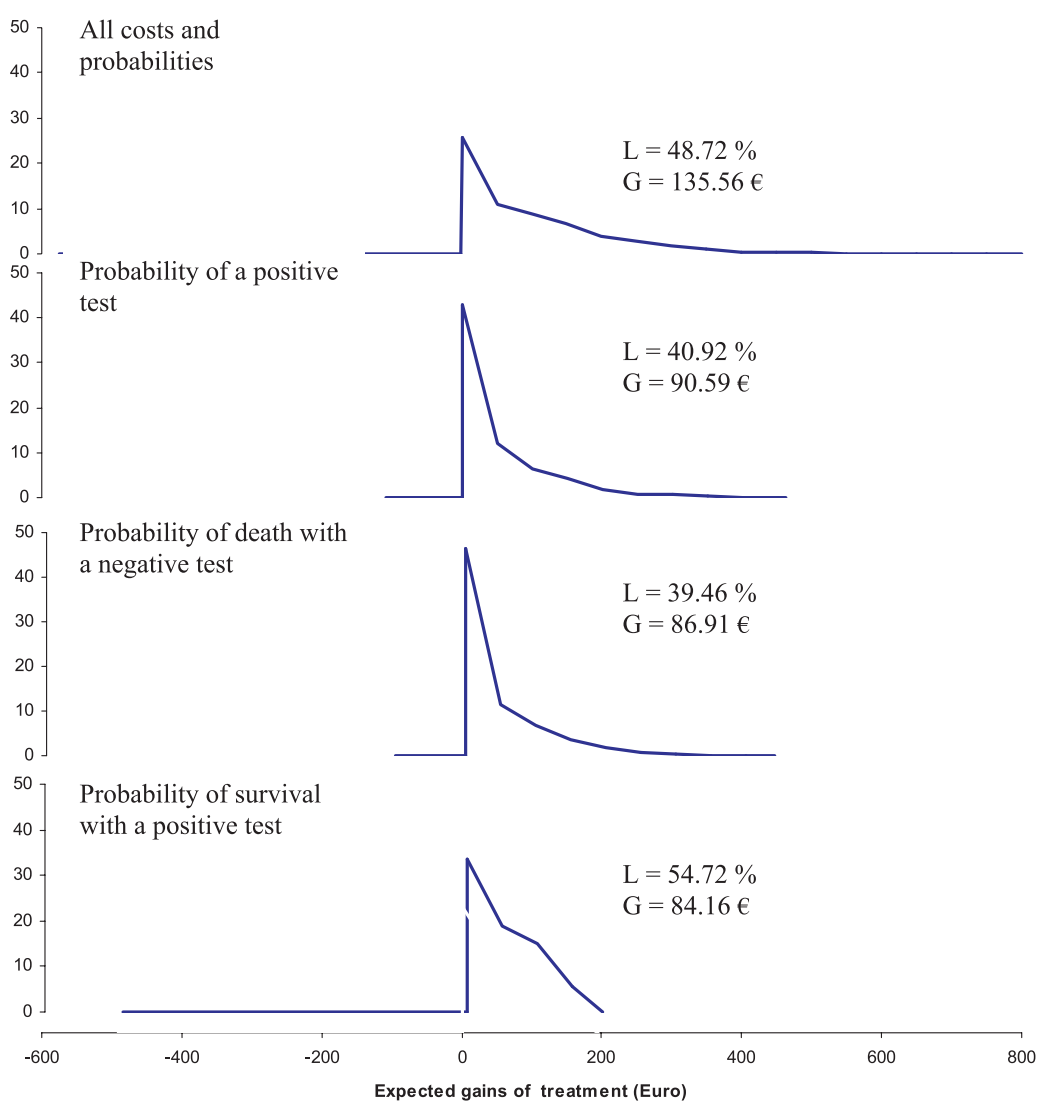

Figure 2. Expected gains of treatment of Belgian White and Blue calves with a positive test (blood lactate $\leq 7.8 \mathrm{mmol} / \mathrm{L}$ ) over no-treatment of calves with a negative test: Javelin diagrams with average expected gains (G) and likelihoods of positive gains (L) associated with random changes in all and in the most influent input variables.

represents the expected extra-gain associated with random changes in the input variables while the left part would represent the extra-loss. The likelihood and the average of this expected extra-gain are represented by the height and the average of the head of the javelin, respectively.

\section{RESULTS}

In the PL study, the test was considered as positive when PL was below some cutoff value, because PL decreased with BRDC severity. The observed expected costs were $\mathrm{EC}_{\mathrm{TRT}}=215.39 €$ and the overall $\mathrm{EC}_{\mathrm{TEST}}=$ $198.01 €$ which indicated that, in this exper- iment, doing the test before deciding on treatment was less costly than directly treating the sick calves. The highest $\mathrm{EC}_{\mathrm{TP}}$ for which $\mathrm{EC}_{\mathrm{TP}}<\mathrm{EC}_{\mathrm{TN}}$ occurred for $\mathrm{PL} \leq 7.8 \mathrm{mmol} / \mathrm{L}$. Indeed, at PL $=7.8 \mathrm{mmol} / \mathrm{L}, \mathrm{p}(\mathrm{S}-\cap \mathrm{T}-)=$ $0.13, \mathrm{p}(\mathrm{S}+)=\mathrm{p}(\mathrm{S}+\cap \mathrm{T}+)=0.77$ which gives $\mathrm{EC}_{\mathrm{TP}}=101.39 €, \mathrm{EC}_{\mathrm{TN}}=108.58 €$ and $\mathrm{EC}_{\text {DIFF }}=108.58-101.39=7.19 €$. Of course, the choice of the cut-off value was driven by the available data and was stained by uncertainty. Consequently, the expected benefit of treatment versus no-treatment could possibly vary with changes in the input variables. This variation is depicted in the javelin diagram (Fig. 2). When all 
input variables are allowed to vary collectively over all six distributions, the minimum $\mathrm{EC}_{\mathrm{DIFF}}$ was $-575 €$, the maximum was $+806 €$, and the average of the positive $\mathrm{EC}_{\text {DIFF }}$ was $135.56 €$, which suggests benefit of treatment of animals with PL $\leq$ $7.8 \mathrm{mmol} / \mathrm{L}$ (over the expected costs of no treatment of negative calves) may increase even further than what was observed with our data. When analyzing the changes in $\mathrm{EC}_{\mathrm{DIFF}}$ by allowing each input variables to vary separately over its own distribution, one observes that $\mathrm{EC}_{\mathrm{DIFF}}$ was very sensitive to changes in the probability of survival with a positive test, in the probability of death with a negative test and in the probability of a positive test (Fig. 2). On the contrary, it was mostly invariant to the economic losses associated with the death of a calf and to the costs of treatment (not shown in Fig. 2). For example, the average of the expected extra-gain associated to random variation in $\mathrm{p}(\mathrm{T}-)$ was $90.59 €$ but only $10 €$ for variation in the animal economic value $\left(\mathrm{C}_{\mathrm{ANL}}\right)$.

In the TV study, the test was considered positive when it was higher than some cutoff value. The observed expected cost for treating the respiratory symptoms with or without knowledge of TV were $\mathrm{EC}_{\mathrm{TEST}}=$ $27.38 €$ and $\mathrm{EC}_{\mathrm{TRT}}=51.55 €$, respectively. Here again, the alternative of doing the test before treatment should be preferred over treatment without knowledge of TV. The highest $\mathrm{EC}_{\mathrm{TP}}$ for which $\mathrm{EC}_{\mathrm{TP}}<\mathrm{EC}_{\mathrm{TN}}$ was $\mathrm{TV}=1.81 \mathrm{~L}$. At this volume, $\mathrm{EC}_{\mathrm{TP}}=7.95 €$ and $\mathrm{EC}_{\mathrm{TN}}=10.26 €$ which gives $\mathrm{EC}_{\mathrm{DIFF}}=$ $2.31 €$. The variation in $\mathrm{EC}_{\mathrm{DIFF}}$ associated with uncertainty in the input variables is depicted in Figure 3. When input variables are allowed to vary collectively, the average positive extra-gain was $24.29 €$ with an extreme minimum and maximum values of $-128 €$ and $+112 €$, respectively. As in the PL study, $\mathrm{EC}_{\text {DIFF }}$ was very sensitive to changes in the probability of death with a negative test, in the probability of survival with a positive test, and in the probability of a positive test. It was also mostly invariant to changes in cost variables.

\section{DISCUSSION}

Because uncertainty is a critical fact about medical reasoning [10,11], veterinarians must often make important decisions based on incomplete knowledge of the probability of survival of a sick animal. The decision to either directly treat the patient or to test it, and at which test values, may be selected in a rational way by decision analysis methods that estimate quantitatively the relative net value of the different options $[10,11]$. As an example, we identified that evaluating PL or TV before treating calves with BRDC was less expensive than directly treating them. Within the limits of our available data, the threshold in Belgian White and Blue calves was at PL $\leq$ $7.8 \mathrm{mmol} / \mathrm{L}$ and at TV $\geq 1.81 \mathrm{~L}$ in Holstein calves. Coghe et al. [3] found that a PL cutoff at $4 \mathrm{mmol} / \mathrm{L}$ is a reliable indicator for mortality within $24 \mathrm{~h}$. However, they obtained the value using a Receiver Operating Characteristic (ROC) curve [4, 7] and they assumed the same clinical worth to false positive and false negative results. However, the costs of false positives (test positive dead calves) were much higher than the costs of false negatives (test negative surviving calves) because they included calf economic values.

Of course, these results should be interpreted carefully since our study has several limitations. Among others, we considered only direct costs of treatment and animal economic values. The analysis covered only a short time-period after treatment and the number of caves was rather small. We assigned no penalty to treatment harms and, because future expected production was unavailable, we used the economic values of the calves at the time of the disease. Last, but not least, we had no information on the probability of survival among healthy and untreated sick calves. It was therefore necessary to perform sensitivity analysis to discover for what variables additional information is required $[5,8]$. In our study, $\mathrm{p}(\mathrm{T}-), \mathrm{p}(\mathrm{S}+\cap \mathrm{T}+)$, and $\mathrm{p}(\mathrm{S}-\cap \mathrm{T}-)$ were more influential than economic variables in the determination of 


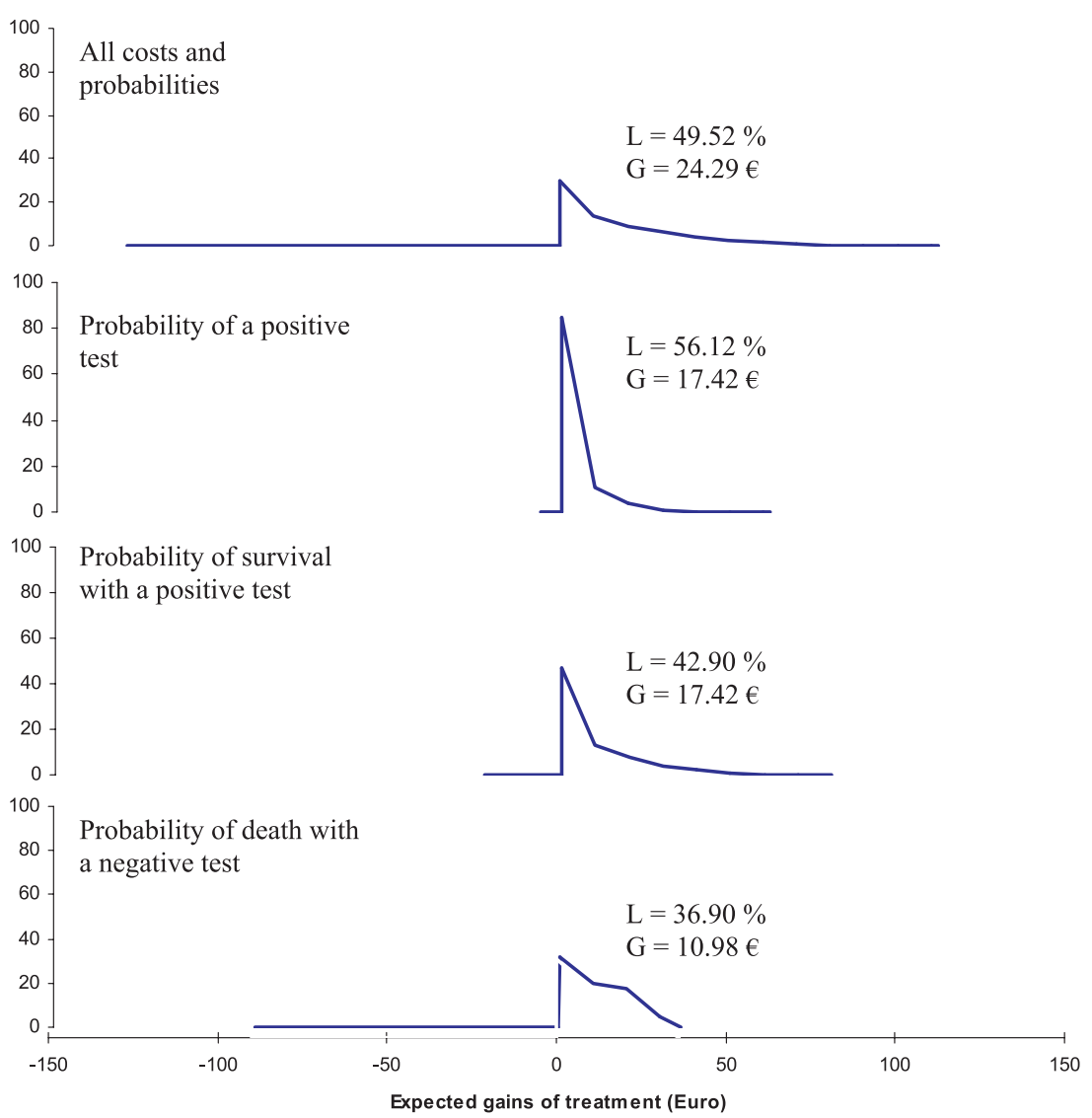

Figure 3. Expected gains of treatment of Holstein calves with a positive test (maximum tidal volume $\geq 1.8 \mathrm{~L}$ ) over no-treatment of calves with a negative test: Javelin diagrams with average expected gains $(\mathrm{G})$ and likelihoods of positive gains associated (L) with random changes in all and in the most influent input variables.

the best alternative. They are the critical uncertainties on which research should focus to have more accurate test threshold in determining animals for which treatment should be the chosen alternative.

Deterministic and probabilistic sensitivity analyses are the two main techniques to analyze uncertainty $[2,8]$. Probabilistic methods are more popular because they take account of both variability and uncertainty in input variables and because they provide a final distribution for the output.
Deterministic analyses give only point estimates of the impact on the decision of uncertainty in the input variables. Their results may be displayed as tornado diagrams that reflect the extreme values in the output associated with extreme values in the input on a variable-by-variable basis. Recently, Felli and Hazen [6] proposed an extension of tornado diagrams, called the javelin diagrams, to display the results of probabilistic sensitivity analyses. For example, in Holsteins (Fig. 3), when all input variables 
are allowed to vary collectively, the lowest and highest values of the overall extra-gain are represented by the left $(-128 €)$ and right $(+112 €)$ extremes of the javelin. The head of the javelin symbolizes the distribution of the extra-gains. The height of the head represents the likelihood of gains $(49.52 \%)$ and the area under it represents the average extra-gain $(24.29 €)$. The javelin diagram also shows the important changes in extragain associated with uncertainty in the different input probabilities. Their individual importance can be readily appreciated by the size of the corresponding javelin heads, the value of the average extra-gains and the likelihood of observing positive extra-gains. This may help in targeting further research directions and in reducing effort in data collection.

Decision analysis methods may also be used to determine how much veterinarians should be willing to pay for a clinical test [5]. For example, at PL $\leq 7.8 \mathrm{mmol} / \mathrm{L}, \mathrm{EC}_{\mathrm{TEST}}=$ $210 €$ and $\mathrm{EC}_{\mathrm{TRT}}=215.39 €$. The difference $215.39 €-210 €=5.39 €$ is the maximum amount a veterinarian should be willing to pay for the PL test. But, while the test was completely specific in the sense that all animals with PL $>7.8 \mathrm{mmol} / \mathrm{L}$ died, it was imperfect since $87 \%$ of the calves with PL $\leq$ $7.8 \mathrm{mmol} / \mathrm{L}$ survived. If it had been totally accurate in identifying both survived and dead animals, the expected cost of performing the test would have decreased further $\left(\mathrm{EC}_{\mathrm{TEST}}=136.04 €\right)$.

\section{ACKNOWLEDGMENTS}

The author would like to thank the scientists from the Department of Physiology, Faculty of Veterinary Medicine, University of Liège (Belgium) for conducting the surveys and collecting the data.

\section{REFERENCES}

[1] Bureau F., Michaux C., Coghe J., Uystepruyst C., Leroy P.L., Lekeux P., Spirometric performance in Belgian Blue calves. II. Analysis of environmental factors and estimation of genetic parameters, J. Anim. Sci. 79 (2001) 1162-1165.

[2] Clemen R.T., Making hard decision: An introduction to decision analysis, Duxury Press, 1996.

[3] Coghe J., Uystepruyst C., Bureau F., Detilleux J., Art T., Lekeux P., Validation and prognostic value of plasma lactate measurement in bovine respiratory disease, Vet. J. 160 (2000) 139-146.

[4] Detilleux J., Arendt J., Lomba F., Leroy P., Methods for estimating areas under receiveroperating characteristic curves: illustration with somatic-cell scores in subclinical intramammary infections, Prev. Vet. Med. 41 (1999) 75-88.

[5] Felli J.C., Hazen G.B., Sensitivity analysis and the expected value of perfect information, Med. Decis. Making 18 (1998) 95-109.

[6] Felli J.C., Hazen G.B., Javelin Diagrams: A graphical tool for probabilistic sensitivity analysis, Decision Analysis [on line], 2003, http://da.pubs.informs.org/AcceptedTitles.htm [consulted the 20 January 2004].

[7] Greiner M., Pfeiffer D., Smith R.D., Principles and practical application of the receiveroperating characteristic analysis for diagnostic tests, Prev. Vet. Med. 45 (2000) 23-41.

[8] Pfeiffer D.U., Decision analysis and risk analysis, in: Ruppanner R. (Ed.), Risk analysis and animal health - A course manual, International training course, Dübendorf, Switzerland, 1997, pp. 861-877.

[9] SAS/IML Software: Usage and Reference: Version 6, first edition, 1996.

[10] Szolovits P., Uncertainty and decisions in medical informatics, Methods Inf. Med. 34 (1995) 111-121.

[11] Tavakoli M., Davies H.T.O., Thompson R., Decision analysis in evidence-based decision making, J. Eval. Clin. Pract. 6 (2000) 111-120. 